\title{
The model of interaction between the enterprise and the state
}

\author{
Mark Vlasov ${ }^{1,}{ }^{*}$, Anna Shekhovtsova ${ }^{1}$, and Tatyana Lelyavina $^{1}$ \\ ${ }^{1}$ Saint Petersburg State University of Architecture and Civil Engineering, Vtoraya Krasnoarmeiskaya \\ str. 4. St. Petersburg, 190005, Russia
}

\begin{abstract}
The purpose of the article is to determine and formalize the mechanism of interaction between an enterprise and the state. The assessment of not even the effectiveness, but the appropriateness of the functioning of the enterprise is in owner's hands, who estimates the amount of income that he can count on when building up an enterprise. The government is also interested in the development and functioning of an enterprise, since the enterprise not only generates income in the form of tax deductions from its income, but also provides employment for the population. Therefore, the state seeks to create the necessary conditions for an enterprise. However, this takes into account a plenty of factors that can provide the company with competitive advantages, thereby contributing to an increase in the income of the owner. The state uses the same factors for taxation, since it believes that the owner should share his income for the possession of the benefits that are provided to him. It is from the point of view of the profitability of the enterprise that the owner considers government policies in the field of economics, finance, credit, and taxes. Therefore, there is a need for a formal definition of the mechanism of interaction between the state and the enterprise in terms of the distribution of income from the latter. The article is addressed, first of all, to persons developing a tax system for assessing the consequences of the adoption of certain decisions in this area. In addition, the article may be of interest to owners of enterprises, investors, economists, as well as representatives of commercial banks.
\end{abstract}

\section{Introduction}

The reasonability of building up an enterprise and its operation is assessed by an owner firstly in terms of the income received. Tax burden, which constitutes a significant part of the income expected by the owner, is given special attention within this assessment. The tax value determines the amount of investments, the location of an enterprise, the production extent and the breadth of a product range. The value of tax burden significantly affects the price of company's products, since the owner, regardless of the location of the company, always relies on certain income, which should not be lower than average income within the industry. This position is peculiar mostly to backbone enterprises that being export-oriented directly or indirectly represent their products on the world market.

\footnotetext{
*Corresponding author: markvlasov@mail.ru
} 
This position affects to a lesser extent backbone enterprises focused on selling their products in local markets, where the tax burden influences equally all represented enterprises. In this case, the tax burden is shifted to the consumer and is also reflected in the salary of personnel at such enterprises.

The staff of backbone enterprises are less affected by the increase in taxes, since wages at such enterprises are usually higher due to:

- higher productivity and less labor intensiveness of products;

- use of high-performance equipment, which is dictated by the scale of manufacture and the desire to reduce costs by attracting more qualified employees.

Moreover, the staff of backbone enterprises constitutes the minority of economically active population of territories where such companies are located.

Backbone enterprises bring the largest share of income to all state budgets, which reasons the interest of authorities at all levels in their activities. In addition to large income, backbone enterprises increase the employment of economically active population, moreover, the profit that the state gets from such company's activities contributes to the development of the social sector, thereby ensuring social stability.

By regulating the value of taxes, the state seeks to attract backbone enterprises by creating the best conditions for business and providing all kinds of benefits, including tax allowances.

Thus, the security of an enterprise largely depends on the position taken by the state, including all levels of legislative and executive power.

The state, trying to create conditions demanded by the enterprise, should take into account a lot of factors that can provide competitive advantages, thereby contributing to the increase in the owner's income. However, the state uses the same factors for taxation, since the authorities believe that owner should share his income with them in return for having the benefits provided by the government. The main factors include a territory and its location, the availability of necessary resources, the proximity of markets, the availability of labor and its competence, skills, traditions, as well as the level of infrastructure development and the external effects. These effects can include [1]:

- negative, mainly associated with the increase in anthropogenic load on a territory and environmental impacts;

- positive, associated with the multiplier effect, stimulating building up more enterprises, that would directly or indirectly interact with the initial one.

Depending on the characteristics of products and the technology of its manufacture, these factors have different effects on the efficiency of an enterprise. Thus, an owner considers the state policy in field of economics, finance, loans and taxes specifically in terms of the income from activities of an enterprise.

\section{Materials and Methods}

Taking into account the point of the state, the owner has to structure his investments to extract the maximum benefit from the conditions provided by the tax system. Thus, both the owner and the state are equally interested in increasing the income of the enterprise, yet when distributing its income, their viewpoints are opposite. The solution of this situation demands a compromise that can be achieved with the help of mathematical modeling. If assuming that the state expects the proposed tax system to be attractive for the enterprise owner, then all that is left to do for the owner is to verify this expectation. The verification consists in determining the value of the owner's income after taxes. The income value itself does not determine anything, since it should be compared with alternative options that the owner has. In this case not only the income value is important, but also the risk that 
accompanies the earning of this income. The decision made by the owner is always subjective, since the ratio of the income value and its risk cannot be objectively established.

Therefore, the state policy in economics, finance, loans and taxes is analyzed and estimated in terms of the income gained from the activities of the enterprise that the owner can reckon on.

It is advisable to present the mechanism of interaction between the enterprise and the state in the form of a mathematical model built on a number of assumptions. The models should reflect the opportunities provided to the enterprise by the state tax system. First of all, it is assumed that the products manufactured by the enterprise take into account such technical and economic indicators that are counted in a prime cost and taxation, such as labor, material, capital, energy, and others. Moreover, this assumption applies not only to a product as a result of production, but also to its consumer attracting properties. Since the same products of the enterprise can be sold in different strategic business areas, the assumption is made that each strategic business area has its own system of taxation of products. This circumstance also takes into account that [2]:

- product requirements may vary for each market segment;

- certain market segment may have its own tax system and customs duties.

As for the technological equipment of the enterprise, it can be assumed that it must comply not only with all the requirements of the legislation in force at the territory where the enterprise is located, but also with all trends contained in this legislation. It concerns depreciation, environmental, social and other policies reflected in the tax system of the location of the enterprise. For example, the legislation may show an interest in increasing employment, backed by collateral allowances, or oppositely in increasing labor productivity, which stimulates a reduction in the labor intensity of products.

Thus, if assuming that at the stages of designing products and launching their production, as well as at the stages of enterprise design and implementation of design solutions, the benefits and allowances provided by tax legislation are already taken into account, in relation to both manufacture of products and their sale. The only variable that takes into account the opportunity to use the provided allowances is the variation in output values, if there is no price limit. When the opportunity to raise the price occurs, the company will immediately take it. One product sold in a specific strategic business area will always be more profitable than another in terms of production and tax legislation, despite the existing difference in prices due to differences in labor, capital, material and energy intensity, as well as the degree of infrastructure development and security resources.

\section{Results}

To develop a model for assessing the influence of tax burden's value and structure on the income of the enterprise, the owner uses the following data. First of all, the set of manufactured products $I$ and the set of strategic business areas $S$, were these products can be sold, are defined. The set of price elements $J$ is also defined. For each strategic business area $\forall s \in S$, vector $\delta_{s}=\left\{\delta_{s, j}\right\}$ is given, each component of which determines the value of tax and customs rates for the price element of the enterprise's products. For each component of vector $\delta_{s, j}$ there is a corresponding total value of tax and customs rates conforming with the conditions of production and sale in strategic business area $s$.

For each type of product $\forall i \in \mathrm{I}$, a vector is given that characterizes the price structure $a_{i}$ $=\left\{a_{i, j}\right\}$, where $j$ is the index of the price element, and $a_{i, j}$ is the value of the price element $j$ of product $i$ in value terms. The sum of the elements is the price of the product, i.e. $c_{i}=$ $\sum_{j \in J} a_{i, j}$.

For each type of product $\forall i \in I$ and each strategic business area $\forall s \in S$ the following terms are given: 
- the interval of the output volume of product $i$ that can be sold in the strategic business area $s\left[n_{i, s}^{\min }, n_{i, s}^{\max }\right]$

- for the boundaries of the interval of product output $i$, the probabilities of the realization of these output volumes $\left[p_{i, s}^{\max }, p_{i, s}^{\min }\right]$ in the strategic business area $s$ are determined, where the first probability corresponds to the lower boundary of the interval of output.

Tax payments for each unit of product $\forall i \in I$ in the strategic business area $\forall s \in S$ are

$$
f_{i, s}=\sum_{j \in J} a_{i, j} \delta_{s, j}
$$

Then, by varying the nomenclature (types of products) and sale volumes in different strategic business areas, under the same output volume in cost terms, it is possible to reduce the tax burden of the enterprise and thereby increase the profit of the enterprise and the income of the owner. If the volume of sales of products of enterprise $B$ is specified, then it is necessary to determine the values of the set of elements $\left\{x_{i, s}\right\}$, so that the composite function characterizing the volume of revenue minus tax payments, i.e.

$$
F=\sum_{i \in I} \sum_{s \in S} p_{i, s} x_{i, s}\left(c_{i}-f_{i, s}\right) \rightarrow \max
$$

reaches its maximum under the restrictions below.

Here

$x_{i, s}$ is the sales volume of product $i$ in the strategic business area $s$;

$p_{i, s}$ is the probability of sales of the volume $x_{i, s}$ of product $i \in I$ in the strategic business area $s \in S$. This probability is determined from the relation

$$
p_{i, s}=p_{i, s}^{\max }-\left(p_{i, s}^{\max }-p_{i, s}^{\min }\right) \frac{\left(n_{i, s}^{\max }-x_{i, s}\right)}{\left(n_{i, s}^{\max }-n_{i, s}^{\text {min }}\right)}
$$

The following restrictions are imposed on the values of variables of the model $\left\{x_{i, s}\right\}$

- the total output of all types of products should not exceed a given value $B$, i.e.

$$
\sum_{i \in I} c_{i} \sum_{s \in S} x_{i, s} \leq B
$$

- the sales volume of each type of product in each strategic business area should be within specified limits, i.e.

$$
n_{i, s}^{\min } \leq x_{i, s} \leq n_{i, s}^{\max }
$$

The presented model allows assessing the prospects of a particular strategic business area when it is impossible to use price competition. However, if in any strategic business area there is an opportunity to increase the price for products, then the situation can dramatically change taking into account the new relationships between price, profit and tax payments. Then, the priority of the pair (products, strategic business area) can be obtained by ranking the values

$$
R_{i, s}=\frac{c_{i}-f_{i, s}}{c_{i}} \text { for } \forall i \in I \text { and } \forall s \in S
$$

If the price changes do not cause the change of the order in the ranked row, then the priorities remain unchanged. The ranking results can be presented in the form of growth share or McKinsey matrices.

\section{Discussion}

Tax payments are focused on the structure of product prices. Any product has its own relationship between labor and capital intensity, material and energy intensity. These relationship is reflected in both cost and tax structures. Therefore, different types of 
products, as well as products of different enterprises will significantly differ because of the conditions under which the company is. If taking into account that enterprises as legal entities are residents of different countries having different tax policies, and that these enterprises sell their products in different strategic business areas, it becomes clear that the priorities of the products have significant differences due to the diversity of current tax regimes [3]. Therefore, in the same strategic business area, the same products of different enterprises will have different prices, competitiveness, profitability and other economic indicators. Thus, when attracting backbone enterprises to one or another territory, it is not enough to take into account the preferences that determine the owner's income in terms of minimizing production costs. It is also necessary to take into account the conditions for the sale of products, including tax and customs regimes of those strategic business areas that the company is interested in.

\section{$5 \quad$ Theoretical and Practical Implications}

The literature widely represents the state's point of view on the goals and objectives of tax policy. It is declared that the purpose of the tax policy is to increase the level of economic development, revitalize business activity, increase employment, stimulate economic activity and eliminate imbalances.

It is assumed that taxes simultaneously fulfill four main functions: fiscal, social (distribution), regulatory and controlling [4]:

1) the main function of taxation is fiscal, since taxes are the predominant component of state budget revenues. The implementation of this function is carried out due to tax control and tax sanctions, which ensure the maximum achievable tax collection and create obstacles for tax evasion. This function implements the main purpose of taxes: the formation and mobilization of financial resources of the state. All other functions of taxation are derived from this function;

2) the social function of taxation consists in the redistribution of public income in favor of weaker and unprotected categories of citizens by placing a tax burden on the stronger categories of the population;

3 ) the regulatory function of taxation is designed to solve the tasks of the economic policy of the state through tax mechanisms. Within the framework of this function, three local taxation functions are distinguished: stimulating, discouraging and reproductive:

- the stimulating local tax function is aimed at creating conditions for building up and developing enterprises that can make the most efficient use of resources of a territory. The purpose of the local function is to increase incomes and employment. Its implementation consists in developing such tax rates and a system of allowances and exemptions that would ensure the most complete use of the territory's resources;

- the discouraging local taxation function is aimed at creating obstacles for enterprises, if their activities negatively affect the economic, social, environmental situation in the territory; if resources for their development are no longer available; resources that are necessary for the creation and development of more promising and effective areas of economic activity are diverted;

- the reproductive local function is designed to accumulate funds for the restoration of renewable resources (primarily labor force) and to compensate for damage from the expenditure of non-renewable resources (for example, mineral resources);

4) the control function of taxation is aimed at timely and complete receipt of tax deductions.

These functions are considered only from the state's point of view, although the performance of these functions is entrusted to enterprises that are run by owners, who assess the possible limits of tax deductions in terms of their income. If owners conclude 
that they bear losses because of the high tax burden, they either transfer their enterprises to a more favorable territory or close them, considering them unprofitable. Therefore, the proposed model is a perfect tool for any company to evaluate the effectiveness of tax policy from the point of view of both the state and the owner of an enterprise.

First of all, this concerns the fiscal function, since tax rates can be so high that backbone enterprises leave the territory, because their activity is considered non-profitable.

The same applies to the regulatory function, the local components of which may not achieve their goals, due to the lack of the stimulating function or the high cost of the reproducing function.

The proposed model fully applies to assessing the adequacy of the tax burden, which is understood as the ratio of taxes and deductions, the real payer of which is an enterprise, to the amount of profit of the enterprise. Applying calculations to the model, the point where tax evasion begins can be determined. The territory, where the owner relocates his enterprise if excessive tax burden appears, can serve as the orienting point for determination.

The variety of applied tax policy models (Fig. 1) also needs to be assessed by the owner for the effectiveness, since such concepts as maximum, minimum, and average tax burden need to be specified and closely linked with other criteria, which the owner goes by. These criteria include economic stability, risks of economic activity and many others [5].

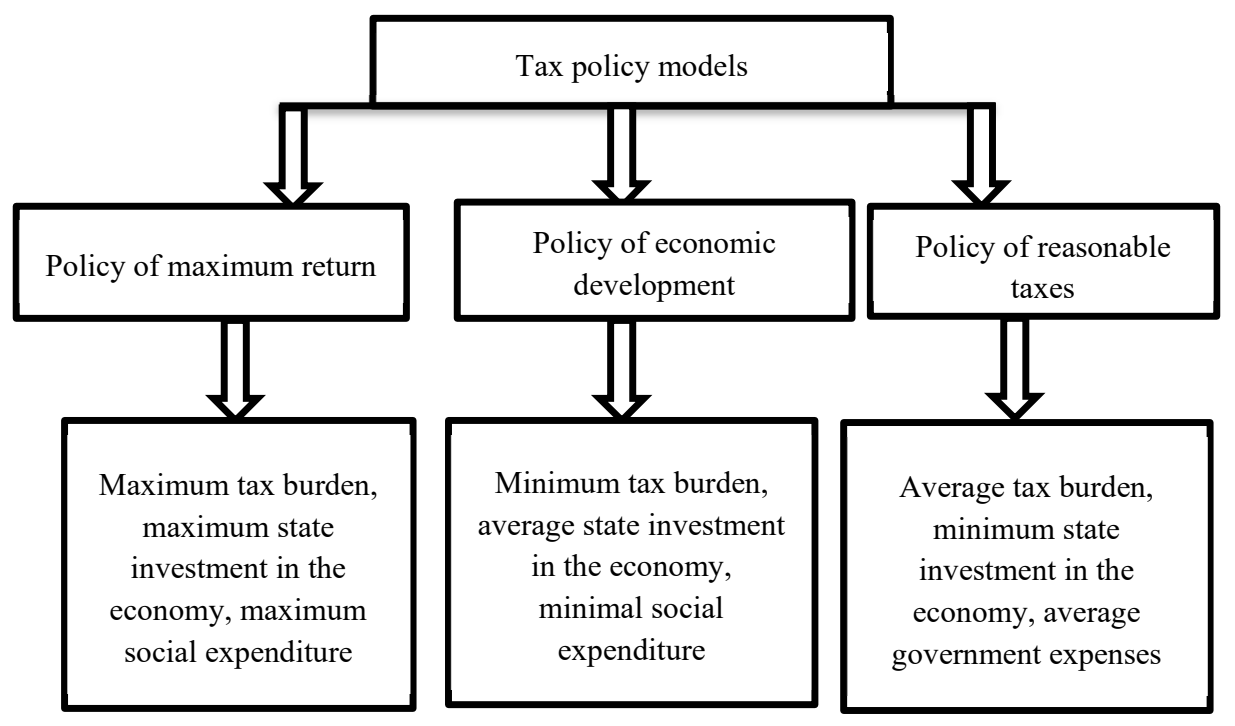

Fig. 1. Tax policy models

Therefore, in terms of practice, the proposed model allows assessing both the existing tax policy and the changes that are constantly proposed by lawmakers. Moreover, any changes directly affect the welfare of the population of the territory and the behavior of owners, which instantly affects business activity, primarily of backbone enterprises. Such enterprises easily change their location if there is a significant threat to the income, unless their activity is related to the extraction of mineral resources. Constraints applied to them cannot be long-term, since they have no prospects. The reduction in investment inflows, termination of updating the product line and subsequent decline in competitiveness will denote the owner's loss of interest in enterprise's activities. In this regard, backbone enterprises may be subjected to greater constraints due to the uniqueness of the market where they can operate. The economic and social aims of the state tax policy in terms of 
eliminating imbalances in social reproduction, stimulating the investment in physical capital increasing and in the development of human capital, apply to all sectors of the economy, including small and medium-scale businesses. The owners of large enterprises, using the possibilities of tax mechanisms to change and expand production activities, introduce advanced breakthrough production management methods, restore and create production ties with large and medium-scale enterprises in different regions, which leads to the acceleration of socio-economic development of regions, increases the level of entrepreneurial activity, contributes to the redistribution of national income and GDP and to the increase in tax deductions. The introduction of methods to increase the efficiency of production management based on cost rationalization can significantly change the structure of product price, and accordingly the structure of tax payments. This is mostly obvious in cases where almost similar sets of tools are used by the entire chain of enterprises that make up the full production cycle, if it is possible [6]. As a result, the conditions for business within strategic business areas are significantly changing.

The government is interested in increasing the number of manufacturing enterprises having strong business ties in various regions, since there is a task of smoothing inequalities in income levels of various population groups and increasing their social security. The increase in the level of income and employment of the population, in its turn, leads to the increase in its level of solvency and, consequently, to the increase in the demand for products. Since the level of solvency of the population is quite low, as well as the growth rate of the number of large and medium-scale production enterprises, which will remain below average in some regions compared with those with developed production, it is impossible to apply methods of price competition. Moreover, production enterprises face the task to offer products that meet the needs of representatives of regional market segments. This problem is effectively solved by varying the product nomenclature, which at the same time addresses another problem: the issue of increasing the volume of product output and its vending in various strategic economic zones [7].

It is the opportunity for the owner to increase sales, reduce the tax burden, raise the profit for the enterprise and the income for himself.

Rationalization of costs and variations of nomenclature positions allow the manufacturer to regulate the cost of product output by offering the product in various strategic economic zones, thereby changing the production interval and the boundaries of probability interval of the output volume of products that can be sold in the strategic economic zone, and to change the structure of the tax burden.

Thus, the model presented in the paper allows solving a number of problems related to the optimization of the actual tax burden, enables rationalizing production capacities, reducing the burden and taking advantage of the incentives implied by the tax system.

\section{References}

1. U.U. Dubina, Beneficiary 14, 31-34 (2017)

2. A.A. Bolatayeva, Scientific News 6, 41-45 (2017)

3. A.M. Grinkevich, Formation of an efficiently fair system of taxation of small businesses (Tomsk, TGU, 2014)

4. I.A. Mayburova, U.B. Ivanova, Tax incentives: theory and practice of application (Moskva: Uniti, 2014)

5. S.A. Voronin, N.Sh. Mamatkulov, R.S. Voronin, Finance 4, 74-78 (2016)

6. I.A. Korostelkina, International Accounting 32, 41-51 (2014)

7. O. M. Nikulina, Finance and Credit 17, 13-27 (2016) 